\title{
An Efficient Low-Power MAC Protocol for Wireless Body Sensor Networks
}

\author{
Daeun Yu and Namgi Kim ${ }^{1}$ \\ Department of Computer Science, Kyonggi University \\ deyoo,ngkim\}@kgu.ac.kr
}

\begin{abstract}
The battery of a sensor node in a wireless body sensor network is small, and therefore has a short lifetime. In consequence, intensive research is being conducted on schemes to efficiently utilize the energy of a sensor node. This paper proposes a new scheme to combine a transmission power control (TPC) model, which is a technology used to heighten energy efficiency in existing body sensor networks, with a low power medium access control (MAC) protocol. This model integrates the short preamble scheme of the existing X-MAC protocol and the TPC model, by combining an early ACK packet and a transmission power control packet. Our scheme efficiently transmits data by predicting the appropriate transmission power before transmitting data packets. We experiment with a TPC algorithm, and analyze the result in order to evaluate the performance of the proposed scheme.
\end{abstract}

Keywords: transmission power control, wireless body sensor system, transmission power control, low power MAC protocol, energy consumption

\section{Introduction}

In modern society, increased human lifespan has led to heightened interest in personal health, and accordingly devices for health management are becoming more widespread [1]. These devices periodically measure body information for patients and elderly people, monitor their body condition, and measure health condition changes in their ordinary life [2]. Wearable devices to monitor health conditions in ordinary life have wireless body sensors [3]. The sensors used for wearable devices are small in size. Sensor batteries are also small, have a short lifetime, and are difficult to replace. As a result, it is very important to increase battery lifetime by efficiently utilizing energy in wireless body sensor networks (WBSN).

Technologies to efficiently utilize energy in wireless body sensor networks are largely divided into the transmission power control (TPC) model, and the low power medium access control (MAC) protocol. Under the TPC model, a sensor node transmits data through an appropriate transmission power level (TPL), thereby saving energy, and a sensor node uses a control packet to notify a sink node of the TPL considered appropriate under the present channel condition [4]. Under such a TPC model, when high transmission power is used while data is being transmitted by a sensor node, much energy is consumed, and low transmission power may heighten the data loss rate. Therefore, it is important to conduct data transmission by finding the appropriate transmission power through a transmission power control (TPC) algorithm. Representative TPC algorithms include linear, binary, and dynamic TPC algorithms.

Under the low power MAC protocol, the device maintains an active state only when it sends and receives data, and otherwise remains in sleep state [5]. In sleep state, the device

${ }^{1}$ Corresponding author: Namgi Kim 
saves energy by operating with minimal functions. A low power MAC protocol has two methods: a synchronous method that synchronizes time among devices and maintains an active state in the same time frame, and an asynchronous method that does not synchronize time, but utilizes a preamble scheme. Under the synchronous method, data is sent and received while an active state is maintained within the determined time frame, and therefore the energy consumption of control packets is small; but it has the demerit of having to maintain active state when there is no data to send or receive. The asynchronous method may overcome such disadvantage, because a sender node uses preambles to awaken the receiver node only when there is data to transmit. When the receiver node is awakened using a preamble by a sender node, the existing asynchronous low power MAC protocol utilizes a long preamble scheme, which triggers high energy waste. As a MAC protocol to compensate for such demerit, Reference [18] proposed an X-MAC protocol [18]. An X-MAC protocol uses a short preamble, and can save greater energy than a scheme utilizing a long preamble.

This paper proposes a new power saving MAC protocol that more efficiently saves energy by integrating the TPC model and the low power MAC protocol, two technologies widely used for energy efficiency in existing wireless body sensor networks. The proposed protocol achieves a more integrated power saving by grafting an X-MAC protocol using a short preamble among lower power MAC protocols onto a TPC model.

\section{Related Work}

\subsection{Transmission Power Control Model}

In a wireless body sensor network, a sensor node transmits collects sensing data to a sink node. Here, the sink node measures the received signal strength indication (RSSI) based on this value, and determines whether to maintain or renew the transmission power of the sensor node [6].

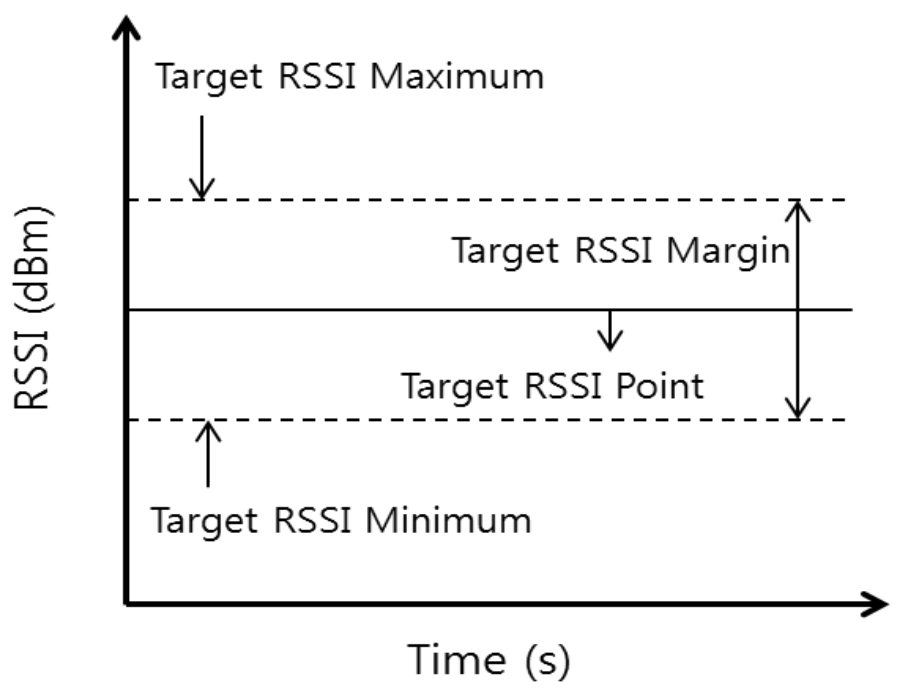

Figure 1. Transmission Power Control Model

In Figure 1, the target RSSI point is an RSSI targeted by the sink node receiving a data packet. The target RSSI margin is a range that adds or subtracts a certain value to the target RSSI point as standard. TPL changing whenever RSSI deviates from the target RSSI point consumes excessive control packets. Therefore, through a target RSSI margin, we reduce waste of the control packets by maintaining the existing TPL when the received packet's RSSI is within range. 
When data packets are transmitted, increasing the transmission power in packet transmission increases the RSSI measured by a sink node as well; and when transmission power lowers, the RSSI also lowers. When the transmission power is high, data may be stably transmitted, but much energy is consumed. On the other hand, when transmission power is low, not much energy is consumed, but the drop rate of data packets increases. In addition, the greater the target RSSI margin, the less control packets that are consumed; but swift adaptation to a changing environment is difficult, which disables the quick saving of energy during data packet transmission. In contrast, a narrower range of margin enables a more sensitive response to the changing environment through swift adaptation. However, the disadvantage is that it requires continuous efforts for RSSI within the margin, consuming many control packets, with the control packet transmission energy becoming high.

The wireless body sensor network employs a closed loop mechanism in order to utilize the TPC model. The closed loop mechanism is a transmission power control method, whereby when a sensor node transmits a data packet to a sink node, the sink node receives the packet, then measures the RSSI, and based on the value, predicts the appropriate transmission power and delivers it to the sensor node through a control packet. When the measured RSSI is within the target RSSI margin, the sink node waits for the next data packet, without sending a control packet. If the RSSI of the received data packet goes beyond the target RSSI margin, the sink node derives a new TPL using the TPC algorithm. Then the sink node includes the newly derived TPL in the control packet, and transmits it to the sensor node. The sensor node that received the control packet transmits the next data packet with new transmission power.

Representative TPC algorithms include linear, binary, and dynamic TPC algorithms. The linear TPC algorithm increases or decreases transmission power by each step, and finds the appropriate transmission power [7]. Although this algorithm is the simplest algorithm, it changes by each step, and therefore consumes much time and many control packets, until finding the appropriate transmission power. The binary TPC algorithm exponentially increases or decreases the transmission power [8]. This algorithm reaches appropriate transmission power more swiftly than the linear TPC algorithm, but substantially changes TPL each time, and therefore an oscillation phenomenon occurs where whenever TPL changes, the RSSI value of the data packet does not belong to the target RSSI margin, and circles around it. The dynamic TPC algorithm derives a linear equation using two RSSIs, and predicts the appropriate TPL at one time through it [9]. Therefore, this algorithm may find the proper TPL most swiftly when the wireless channel is stable. However, it has the demerit of sending too many control packets, because it fails to precisely predict the linear equation, resulting from the frequent occurrence of a phenomenon where the RSSI value is low, even though the RSSI value or TPL value is high, despite the low TPL value. Diverse TPC algorithms have been developed to complement such disadvantage [10-12]. In particular, the equation-based TPC (ETPC) algorithm is a method to induce correlation between TPL and the RSSI value with a linear equation existing uniquely according to the characteristics of the radio chip, and predicting an optimal TPL suitable for the current wireless condition [12]. The ETPC algorithm predicts the optimal TPL using correlation between TPL and RSSI, and therefore may infer an optimal TPL value in comparison with other TPC algorithms.

\subsection{Low Power MAC Protocols}

Under the low power MAC protocol, a device alternately maintains active and sleep states for saving energy. The device stays in an active state when receiving or sending data, and otherwise stays in a sleep state. When it is in a sleep state, the device related to communications blocks energy consumption, thereby inducing overall energy saving. As a result, for energy efficiency of the device it is good to increase the time in a sleep state, rather than the time in an active state. However, the opportunity to send and receive data 
among the devices becomes smaller when the active state shortens due to long sleep states. Therefore, much research has to date been conducted on low power MAC aimed at efficiently distributing the active and sleep states [13-14].

The low power MAC protocol is divided into synchronous and asynchronous methods. The synchronous low power MAC protocol gives and receives data by synchronizing the time between devices. Representative synchronous low power MAC protocols include the sensor-MAC (S-MAC) protocol [15], and the timeout-MAC (T-MAC) protocol [16] as shown Figure 2.

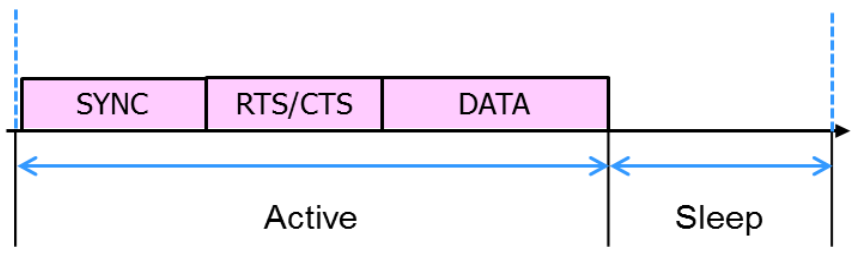

(a) S - MAC Protocol

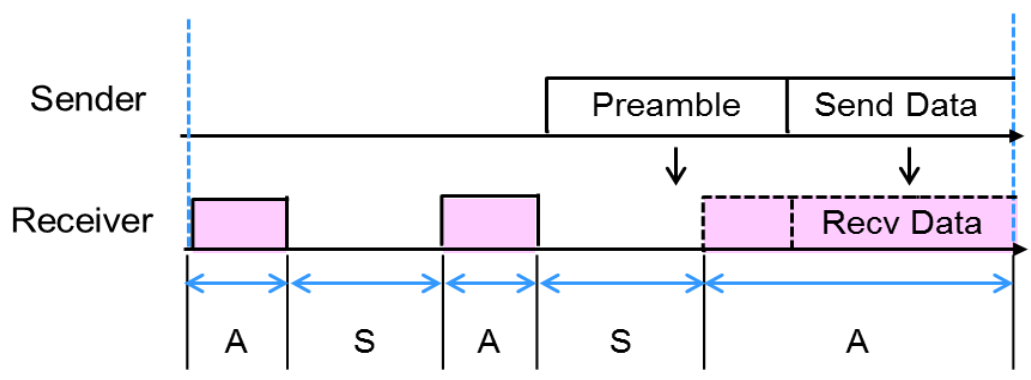

(b) B - MAC Protocol

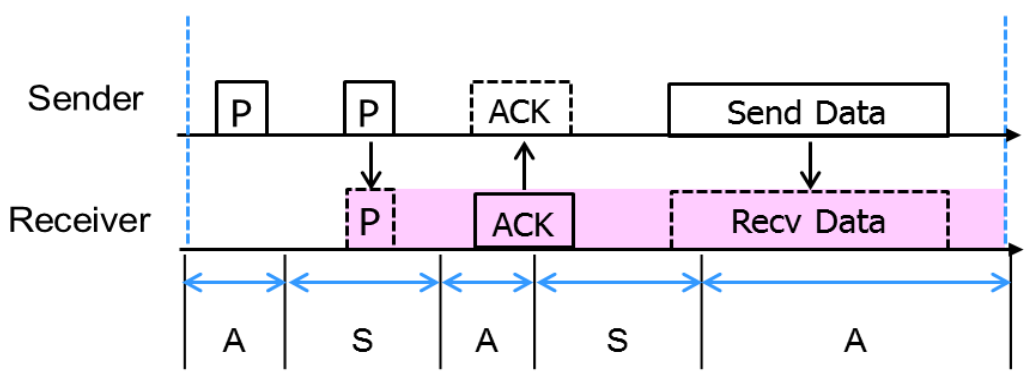

(c) X - MAC Protocol

Figure 2. Low Power MAC Protocols

Under the asynchronous low power MAC protocol, devices do not synchronize time with each other; when there is data to be sent by a sender node, preamble signals are transmitted first, the receiver node moves to an active state, and then data is transmitted. The preamble signal includes information on the destination address where the receiving data is targeted. Representative asynchronous low power MAC protocols using such preamble signals include the Berkeley MAC (B-MAC) protocol [17], and the X-MAC protocol [18]. The B-MAC protocol transmits long preambles to the receiver node first, before the sensor node sends data. The B-MAC protocol utilizes a preamble longer than the sleep time of the receiver node, and even a receiver node in sleep state can hear preamble signals without failure. However, under the B-MAC protocol, even though the receiver node becomes an active state and can receive data, a sensor node should transmit long preambles until the end, and the receiver node should hear it until the end. As a 
result, much energy is wasted. The X-MAC protocol utilizes short preambles, in order to prevent energy waste occurring due to long preambles. Short preambles include destination address information, and continuously transmit such information until the receiver node responds with ACK packets. Therefore, when the X-MAC protocol is used, the sender may save some energy spent for preamble transmission, and the receiver may save some energy spent for preamble receipt. Therefore, this paper proposes a new power saving MAC protocol that may comprehensively save energy by integrating the X-MAC protocol, a short preamble MAC protocol, and a TPC model.

\section{Proposed MAC Protocol}

This paper proposes a new energy-efficient MAC protocol that combines a short preamble MAC protocol and a TPC model in a wireless body sensor network. Under the existing short preamble MAC protocol, an early ACK packet is transmitted to a sensor node in order to notify that a sink node is ready to receive data; under the TPC model, a control packet should be transmitted to a sensor node in order for a sink node to control transmission power. The protocol proposed by this paper integrates the early ACK packet and control packet into one. This paper therefore presents a measure to integrate a short preamble scheme with a TPC model, and operate it. To this end, a sink node adds a transmission power level field to the early ACK packet, and transmits it to the sensor node. In addition, before transmitting a data packet, the sink node transmits a number of short preambles, and uses a TPC algorithm to predict the appropriate transmission power level.

Figure 3 shows a schematic of the proposed MAC protocol. Before a sender node transmits data, it transmits a short preamble to a receiver node. When the receiver node is in sleep state, there is no response; and when the receiver node is in active state, it receives short preambles, and measures the RSSI. Here, when the measured RSSI goes beyond the target RSSI margin, it uses a TPC algorithm to re-predict the appropriate transmission power. Existing X-MAC protocols exchange a short preamble and an early ACK packet once. However, the proposed MAC protocol, in order to find an appropriate TPL according to the TPC algorithm, may exchange a short preamble and an early ACK packet once or more. Nevertheless, excessive exchange between a short preamble and an ACK packet leads to excessive waste of energy, and therefore the maximal number of exchanges should be restricted to the threshold value, and the protocol repeats the exchange until an appropriate TPL is found within the limited range. The process of finding an appropriate TPL is the same as the method proposed by the TPC model. The receiver node transmits a newly calculated TPL in an early ACK packet to a sender node. A sender node updates the received TPL, and utilizes it in the next short preamble transmission. Thereafter, if the RSSI of the short preamble packet received by the receiver node belongs to the target RSSI margin, the receiver node transmits only the last early ACK packet. The sender node that received this transmits the data packet using the present TPL. 


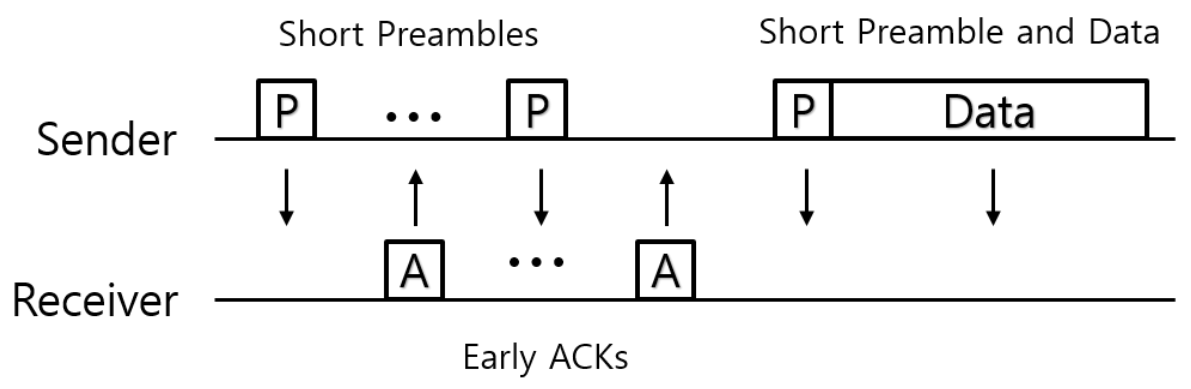

An Early ACK includes the newly calculated TPL by RSSI of the previous short preamble. A short preamble is transmitted with TPL delivered through the previous early ACK.

Figure 3. The Proposed MAC Protocol

\section{Experiments}

\subsection{Experimental Environments}

We conducted this experiment using an actual wireless body sensor. Table 1 shows the experimental equipment and environmental variables. For this experiment, we used a Cricket Mote produced by Crossbow and a CC1000 Radio module [19]. The CC1000 Radio module has a total of 23 stages of transmission power [20]. We set the target RSSI margin at $-85 \mathrm{dBm}$, and when the value was lower than $-120 \mathrm{dBm}$, we considered it a loss. Figure 4 and 5 shows the Cricket Mote and the places at which nodes are attached, respectively. We attached the sensor node transmitting data to the back, and attached the sink node receiving data to the chest.

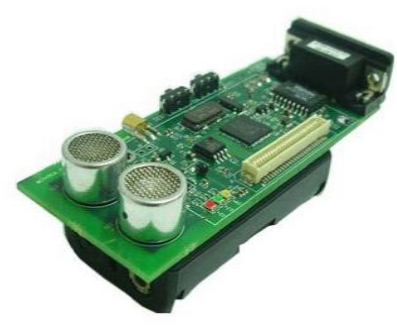

Figure 4. Cricket Mote

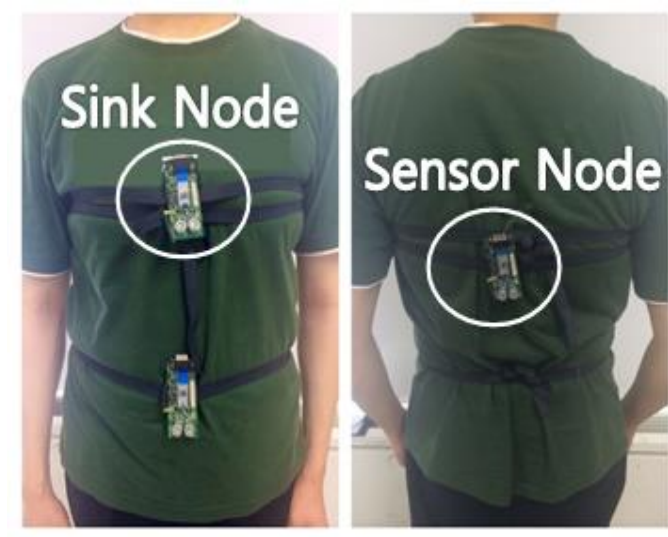

Figure 5. Node Locations

The movement of the experimenter consisted of standing, walking, and running. The experiment was performed indoors and outdoors. In a wireless body sensor network, a sensor node and a sink node exchange data in the units of second, minute, and hour, according to the situation. In this experiment, data was exchanged in the units of one second, and the subject's movement and location could change every time data was sent. We set the maximal number of the short preambles at eight. This experiment examined the result of applying diverse TPC algorithms under a MAC protocol using a short preamble. The TPC algorithms we used in the experiment were MAX, MIN, Linear, Binary, Dynamic, and ETPC. A MAX algorithm refers to a method to transmit TPL without changing it dynamically, and fixing it to maximal power (10 dBm); and MIN algorithm means an algorithm fixing TPL to a minimal power $(-8 \mathrm{dBm})$, and transmitting 
it. Under MAX and MIN algorithms, TPL does not change dynamically, and it is not necessary to transmit a control packet. Figure 6 shows the average RSSI according to body movement and location. In the figure, In denotes Indoor, and Ot denotes Outdoor.

Table 1. Experimental Parameters

\begin{tabular}{|c|c|c|c|}
\hline Sensor properties & Values & Experimental setups & Values \\
\hline Device Model & Cricket Mote & Sensor placement & Back \\
\hline Radio module & CC1000 & Sink placement & Chest \\
\hline Supply voltage & $2.5 \mathrm{~V}$ & Body movements & Standing, \\
\hline Radio frequency & $433 \mathrm{MHz}$ & Body locations & Indoor, Outdoor \\
\hline $\begin{array}{c}\text { Transmission bit } \\
\text { rate }\end{array}$ & $19.2 \mathrm{kbps}$ & Target RSSI point & $-85 \mathrm{dBm}$ \\
\hline Output power & -8 to $10 \mathrm{dBm}$ & Target RSSI margin & $\pm 5 \mathrm{dBm}$ \\
\hline $\begin{array}{c}\text { Transmission } \\
\text { current }\end{array}$ & 8.2 to $26.7 \mathrm{~mA}$ & Packet drop RSSI & $-120 \mathrm{dBm}$ \\
\hline Reception current & $9.3 \mathrm{~mA}$ & $\begin{array}{l}\text { Maximum number } \\
\text { of short preambles }\end{array}$ & 8 \\
\hline $\begin{array}{c}\text { Packet size } \\
\text { preamble size }\end{array}$ & $\begin{array}{l}36 \text { Bytes } \\
4 \text { Bytes }\end{array}$ & $\begin{array}{l}\text { Sensing period } \\
\text { Experiment time }\end{array}$ & $\begin{array}{c}1 \mathrm{~s} \\
300 \mathrm{~s}\end{array}$ \\
\hline
\end{tabular}

\subsection{Experimental Results}

In this experiment, we analyzed the energy consumption amount by each TPC algorithm according to the subject's movements. The movements of the subject consisted of standing, walking, and running. We experimented with alternate indoor and outdoor locations, and transmitted a total of 100 data packets. Figure 7 (a) shows a situation where data was sent and received indoors and outdoors alternately while standing, and Figure 7 (b) and 7 (c) displays a graph of energy consumption while walking and running, respectively. Overall, the standing condition consumed more energy than the other movements. In particular, the transmission energy for short preambles accounts for a large portion of the energy, and Figure 6 shows that the reason is the difference of appropriate transmission power between indoor and outdoor is large while standing. For example, in the case of standing indoors, when a sensor node transmits data with consumption power of $-6 \mathrm{dBm}$, a sink node receives an average of $-85 \mathrm{dBm}$ RSSI. However, when the location changes to outdoors, the sink node receives an average of $-105 \mathrm{dBm}$ RSSI, even though transmission occurs with the same consumption power. Outdoors, a sink node may receive an average of $-85 \mathrm{dBm}$ RSSI only when transmission occurs at about $5 \mathrm{dBm}$. As shown above, the appropriate transmission power differs by more than $10 \mathrm{dBm}$. Therefore, in the case of standing, location changes consume a lot of short preambles in order to find the appropriate transmission power. In particular, a linear TPC algorithm that increases and decreases the transmission power by each step consumes the most energy. On the other hand, Figure 6 shows that in the case of running or walking, the appropriate transmission power does not differ much between indoors and outdoors.

Lastly, the ETPC algorithm largely exhibited the best efficiency among the TPC algorithms. The ETPC algorithm consumed less energy on the short preambles compared to the other algorithms. The reason is that the ETPC algorithm consumed fewer short preambles in all movements unlike the other algorithms, with a lot of changes in the average number of short preambles according to movement. In other words, the ETPC algorithm predicts the next transmission power with a new linear equation whenever the situation changes, and is able to swiftly reach an appropriate TPL using few short preambles. 


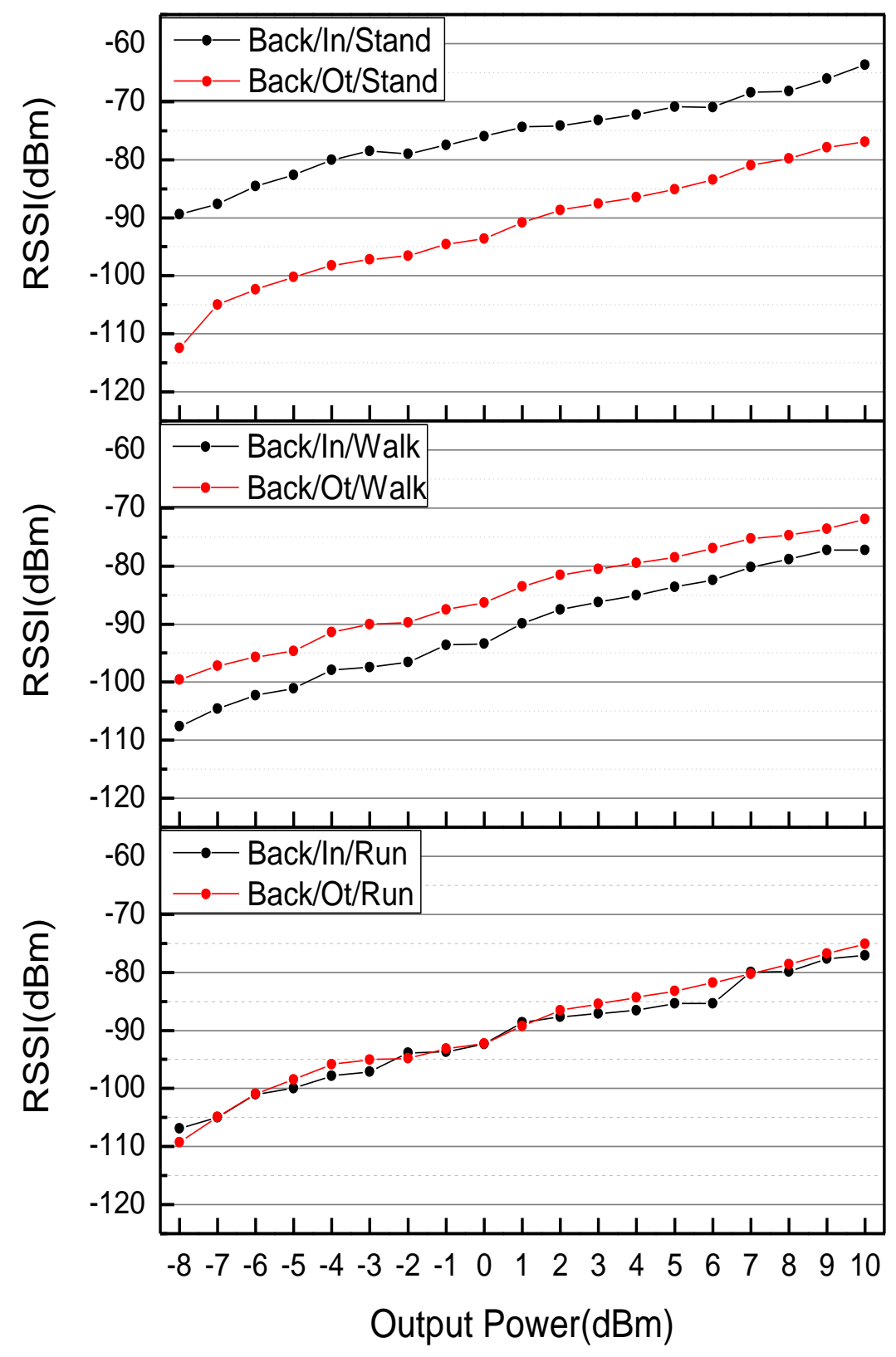

Figure 6. Average RSSI according to Body Movement and Location 

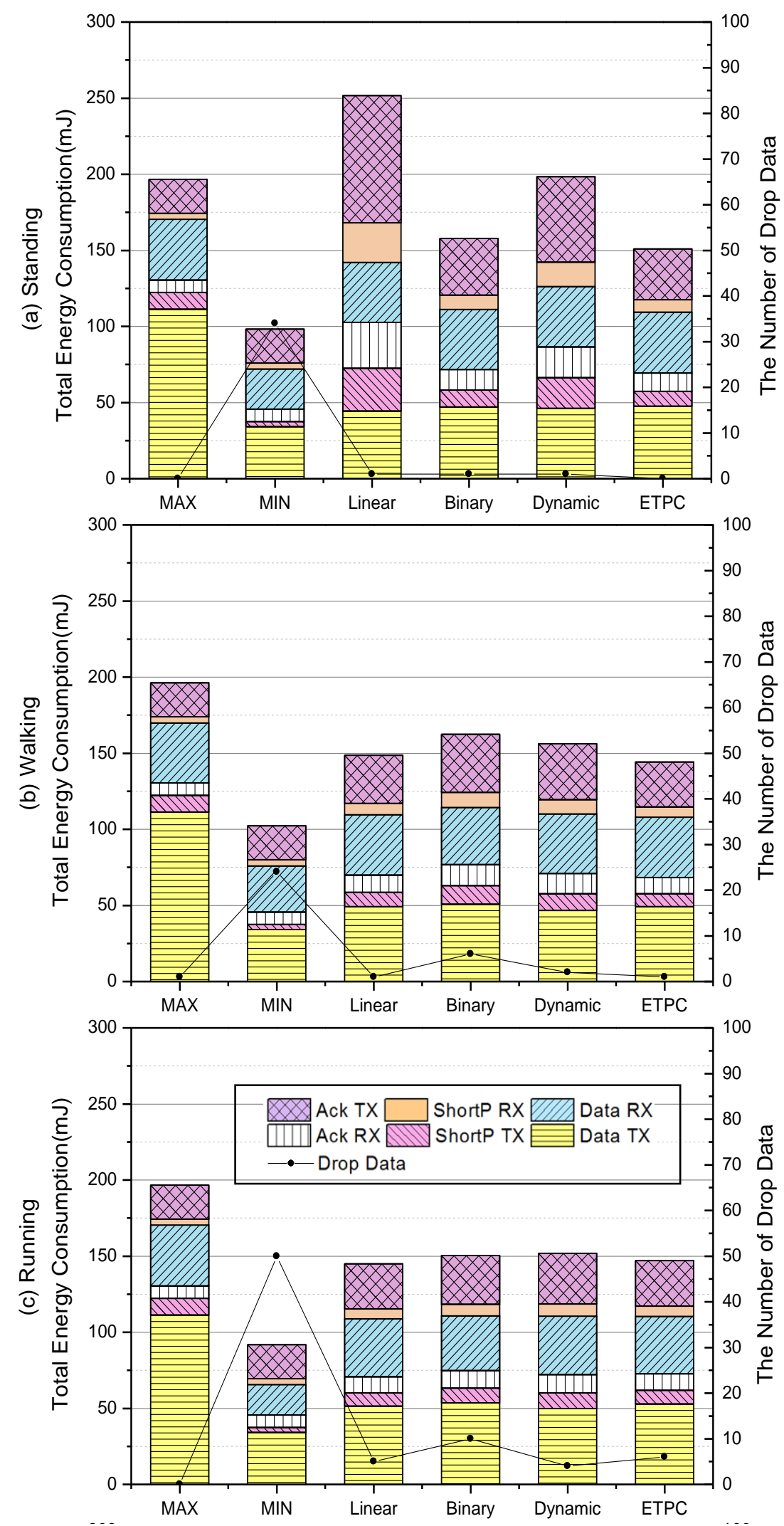

Figure 7. Energy Consumption and Number of Drop Data 


\section{Conclusion}

This paper proposes a new low power MAC protocol for efficient energy management in a wireless body sensor network. We divided an existing sensor device energy saving technology into a low power MAC protocol that saved energy by alternating the device between active and sleep states, and a TPC model that saved energy by controlling the transmission of power transmitting data. In addition, under the lower power MAC protocol, most asynchronous low power MAC protocols use long preambles but an XMAC protocol saves energy by using a short preamble and an early ACK packet.

We propose a new energy-efficient MAC protocol that combines a short preamble MAC protocol like an X-MAC protocol with a TPC model. We combined an early ACK packet and a control packet by adding a new TPL predicted by a TPC algorithm to an early ACK packet of the short preamble MAC protocol. Further, we predicted an appropriate transmission power before transmitting data using a short preamble and an early ACK packet. In addition, using a short preamble whose size was smaller than a data packet and an ACK packet, we efficiently found an appropriate TPL, thereby effectively saving energy for the transmission of a data packet whose size might be larger than a preamble, and reducing the data packet drop rate.

We applied the proposed MAC protocol to different TPC algorithms in order to examine its performance, and conducted comparative analysis through actual experiments. The result was that in the case of experiments according to the subject's movement, the difference in appropriate transmission power between indoors and outdoors while standing was large, with the TPC algorithms consuming many short preambles and ACK packets compared to the other movements. Among the algorithms, the ETPC algorithm consumed lesser energy regardless of changes in movement. The reason is that the ETPC algorithm finds an appropriate TPL with a new linear equation whenever the environment changes, consuming a smaller number of short preambles. We consider future research is needed on protocols to efficiently transmit data, even when the size of data is large.

\section{Acknowledgments}

This work was supported by Kyonggi University Research Grant 2015.

\section{References}

[1] G. V. Crosby, T. Ghosh, R. Murimi and C. A. Chin, "Wireless body area networks for healthcare: a survey", International Journal of Ad hoc, Sensor \& Ubiquitous Computing, vol. 3, no. 3, (2012).

[2] C. Y. Park, J. H. Lim, S. J. Park and S. H. Kim, "Technical Trend of U-Healthcare Standardization", Electronics and Telecommunications Trends, vol. 25, no. 4, (2010)

[3] T. Han, N. Lee, H. Yoon and D. Ryu, "Wearable Computer and Future Information Society", KISE Communications, vol. 18, no. 9, (2000),pp. 21-30.

[4] S. Xiao, "Transmission Power Control in Body Area Sensor Networks for Healthcare Monitoring", IEEE Journal of Selected Areas on Communications, vol. 27, (2009), pp. 37-48.

[5] I. Demirkol, "MAC protocols for wireless sensor networks: a survey", IEEE Communications Magazine, vol. 44, no. 4, (2006), pp. 115-121.

[6] A. Saeed, M. Faezipour and M. Nourani, "A scalable wireless body area network for bio-telemetry", Journal of Information Processing Systems, vol. 5, no. 2, (2009), pp. 77-86.

[7] D. Kunth, "The art of computer programming. 3: sorting and searching", Addison-Wesley, New York, NY, USA, 3rd edition, (1997).

[8] T. H. Cormen, C. E. Leiserson, and R. L. Rivest, "Introduction to algorithms", MIT Press and McGrawHill, 1st edition, (1990).

[9] M. Quwaider, J. Rao and S. Biswas, "Body-posture-based dynamic link power control in wearable sensor networks", IEEE Communications Magazine, vol. 48, no. 7, (2010), pp. 134-142.

[10] L. Zheng, W. Wang and M. Alan, "O'Flynn, Brendan, Hayes, and Michael”, An adaptive transmission power control method for wireless sensor networks, Signals and Systems Conference, (2010), pp. 261265. 
[11] W. S. Lee, B. D. Lee and N. Kim, "Hybrid transmission power control for wireless body sensor systems", International Journal of Distributed Sensor Networks, vol. 2014, (2014).

[12] N. Kim, J. Hong and B. Lee, "Equation-based transmission power control for wearable sensor systems", IEICE Transactions on Fundamentals of Electronics, Communications and Computer Sciences, vol. E98-A, no. 7, (2015), pp. 1558-1561.

[13] A. E. Hoiydi and J. D. Decotignie, "WiseMAC: An ultra-low power MAC protocol for multi-hop wireless sensor networks", Algosensors, (2004), pp. 18-31.

[14] S. Du, A. K. Saha and D. B. Johnson, "RMAC: A routing-enhanced duty-cycle MAC protocol for wireless sensor networks", 26th IEEE International Conference on Computer Communications, (2007), pp. 1478-1486.

[15] W. Ye, J. Heidemann and D. Estrin, “An energy-efficient MAC protocol for wireless sensor networks", Infocom, vol. 3, (2002), pp. 1567-1576.

[16] T. V. Dam and K. Langendoen, "An adaptive energy-efficient MAC protocol for wireless networks", 1st International Conference on Embedded Networked Sensor Systems, (2003) pp. 181-180.

[17] J. Polstre, J. Hill and D. Culeer, "Versatile low power media access for wireless sensor networks", ACM SENSYS, (2004).

[18] X. Buettner, G. V. Yee, E. Anderson and R. Han, "X-MAC: A short preamble MAC protocol for dutycycled wireless sensor network", 4th International Conference on Embedded Networked Sensor Systems, (2006), pp. 307-320.

[19] Crossbow-Technology, http://www.willow.co.uk/Cricket_Datasheet.pdf

[20] CC1000 Data Sheet, http://www.ti.com/lit/ug/swru058/swru058.pdf

\section{Authors}

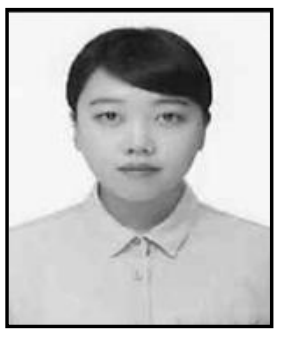

Daeun Yu, she received the B.S. degree in Computer Science from the Kyonggi University, Korea, in 2014. She is currently M.S. candidate in Computer Science from Kyonggi University. Her research interests include wireless systems, sensor networks.

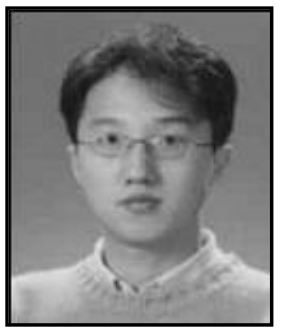

Namgi Kim, he received the B.S. degree in Computer Science from Sogang University, Korea, in 1997, and the M.S. degree and the $\mathrm{Ph} . \mathrm{D}$. degree in Computer Science from KAIST in 2000 and 2005, respectively. From 2005 to 2007, he was a research member of the Samsung Electronics. Since 2007, he has been a faculty of the Kyonggi University. His research interests include sensor system, wireless system, and mobile communication. 
International Journal of Future Generation Communication and Networking Vol. 9, No.7, (2016) 